\title{
Wie riskant sind Antipsychotika für ältere Pflegeheimbewohner?
}

Erkenntnisse aus einer Vielzahl randomisierter kontrollierter Untersuchungen und Verlaufsstudien belegen ein erhöhtes Risiko für die Gesamtmortalität und speziell das Auftreten zerebrovaskulärer Ereignisse sowohl unter atypischen als auch konventionellen Antipsychotika. Aktuelle Erhebungen zeigen, dass bis zu einem Drittel aller älteren Pflegeheimbewohner mit Antipsychotika und von diesen ein nicht geringer Anteil in „Off-label“-Indikation behandelt wird.

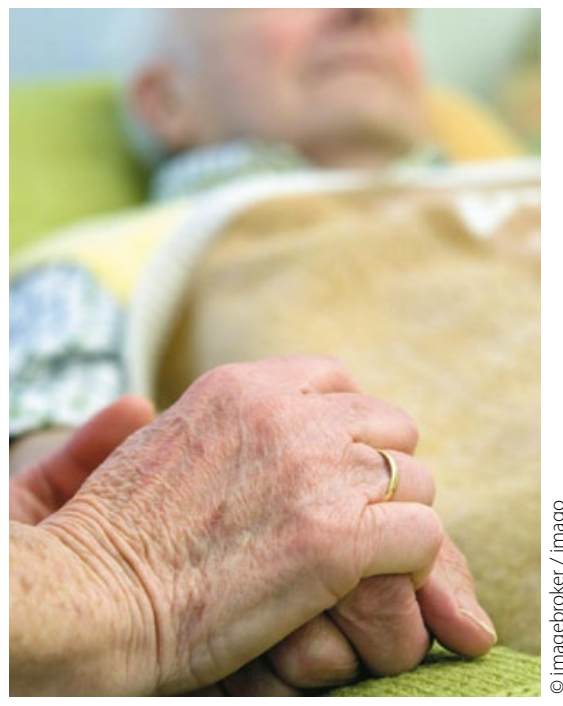

Vorsicht mit Antipsychotika bei Pflegeheimbewohnern.

- In einer populationsbasierten Kohortenstudie wurden 74445 US-amerikanische Pflegeheimbewohner der Jahre 2001 bis 2005 (Alter $\geq 65$ Jahre) mit Neueinstellung auf ein Antipsychotikum erfasst. Für Haloperidol sowie die Atypika Aripiprazol, Olanzapin, Quetiapin und Ziprasidon wurden jeweils über 180 Tage nach Therapiebeginn die Gesamtmortalität sowie einzelne Todesursachen im Vergleich zu Risperidon ermittelt.

In $49 \%$ der Todesfälle lag eine kardiovaskuläre, in $10 \%$ eine zerebrovaskuläre und in $15 \%$ eine respiratorische Ursache vor. Im Vergleich zu Risperidon zeigte sich das (nicht Malignom-assoziierte) Gesamtmortalitätsrisiko für Haloperidol um das Doppelte erhöht. Keine relevanten Unterschiede zu Risperidon ergaben sich für Olanzapin, Ziprasidon und Aripiprazol. Quetiapin wies ein erniedrigtes Gesamtmortalitätsrisiko auf.
Sowohl die Risikoerhöhung bei Haloperidol als auch die Risikoerniedrigung bei Quetiapin zeigten sich auch für sämtliche spezifische Mortalitäten (kardiovaskulär, zerebrovaskulär, respiratorisch, sonstige).

- K. F. Huybrechts et al.

Differential risk of death in older residents in nursing homes prescribed specific antipsychotic drugs: population based cohort study. Brit. Med. J. 344 (2012) e977

\section{Kommentar}

Ein erhöhtes Mortalitätsrisiko unter Antipsychotika besonders in geriatrischen Populationen kann inzwischen als gesichert gelten. Zusammen mit Befunden eher geringer Wirksamkeit von Atypika bei demenzassoziierten Psychosen ist daher diesbezüglich eine besonders strenge Indikationsstellung zu fordern. Die vorliegende Studie zeigt einmal mehr, dass auch bei konventionellen Antipsychotika ein erhöhtes Sterblichkeitsrisiko besteht, und zwar - trotz überwiegend kardio- und zerebrovaskulärer Ätiologien - weitgehend unabhängig von spezifischen Todesursachen.

Im Versorgungsalltag in Pflegeheimen ist eine Tendenz zu beobachten, einmal etablierte antipsychotische Medikationen langfristig unverändert beizubehalten. Die Ergebnisse der vorliegenden Studie mahnen erneut zu einer regelmäßigen Indikationsprüfung und zeigen weiterhin, dass im Fall der fortbestehenden Indikation immer eine Reduktion auf die geringstwirksame Dosis geboten ist.

C. LANGE-AssCheNFELDT =

\section{ACHTUNG:}

\section{Hier muss der Dummy durch eine Anzeige ersetzt werden !!}

